\title{
Safety of direct trocar insertion in various gynecological laparoscopic surgeries
}

\author{
Vipul Patel \\ Assistant Professor, Dept. of Obstetrics and Gynecology, Dr. M. K. Shah Medical College \& Research Center, Ahmedabad, \\ Gujarat, India
}

*Corresponding Author: Vipul Patel

Email: drvipul2112@gmail.com

Received: $30^{\text {th }}$ April, 2018

Accepted: $5^{\text {th }}$ June, 2018

\begin{abstract}
Introduction: To create pneumoperitoneum is the first step to any successful laparoscopic procedure. Various techniques for creation of pneumoperitoneum are traditional veress needle, open method, visual entry system and direct trocar insertion (DTI). Despite of rapid increase in the number of laparoscopic surgeries every year there is no clear guideline on the safe method of entry into the abdomen.

Objective: The aim of this study is to evaluate safety and complications of direct trocar insertion.

Materials and Methods: Retrospective multicenters study of patients who were undergoing various gynecological laparoscopic surgeries. All patients were undergoing DTI access method for primary port insertion. The study focuses on effectiveness of BMI, parity, previous surgery for DTI and evaluates complications.
\end{abstract}

Conclusion: DTI is safe, fast and reliable alternative to primary peritoneal access and creation of pneumoperitoneum.

Keywords: Laparoscopy, DTI (direct trocar insertion), Veress needle, Pneumoperitoneum.

\section{Introduction}

The critical step of laparoscopic surgery is the creation and maintenance of a robust and safe peritoneal operative compartment. ${ }^{1}$ Successful establishment of secure abdominal ports needs three fundamental principles of port creation: access method, access instrument and access location. Failure or success of laparoscopic surgery depends on these three parameters.

Peritoneal port insertion is associated with risk of intra-peritoneal and retro-peritoneal structural damage and most of these serious complications occurred before intended surgery. ${ }^{1}$

Primary peritoneal access methods can be classified as follows.

\section{Chart 1}

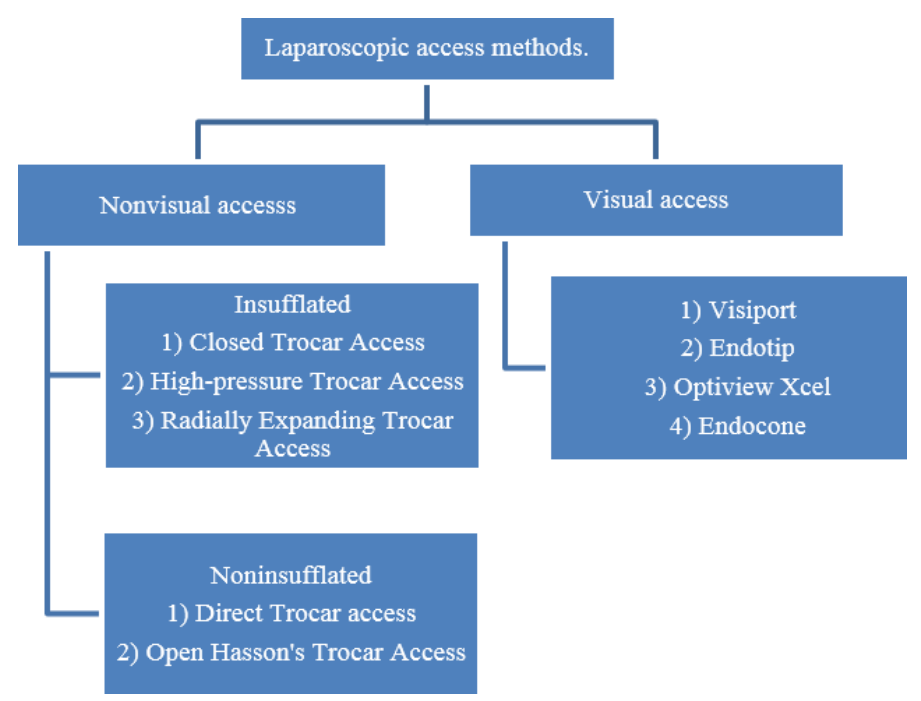

Despite of proper surgical technique and performance, complications can occur in all of these methods.

Most commonly performed conventional closed method used veress needle to distend the virtual peritoneal cavity with co 2 gas and then push through trocar and cannula was inserted to establish a primary laparoscopic access port. It is believed that veress insertion is safer, but various complications were reported, such as injury to vessels, perforation and insufflations of bowel, subcutaneous or subfascial insufflations and gas embolism. 
Hasson in 1971 described open access method with no vascular injury, gas embolism, extraperitoneal insufflations but does not reduce bowel injury and it is associated with gas leak and port instability. ${ }^{2}$

In 1978 Dingfelder first published on direct trocar insertion in a non insufflated peritoneum for laparoscopic surgery. ${ }^{3}$ Various advantages of this method are elimination of veress needle related complications: unsuccessful insufflations, preperitoneal or visceral insufflations and most serious gas embolism. ${ }^{4}$ Direct trocar insertion is one step blind procedure as compared to the closed method with verses which has three blind steps: veress insertion, insufflations and trocar insertion. As DTI is a direct method for peritoneal access it is most rapid method compared to other available techniques, and it is the least performed entry method till today. ${ }^{5}$

Several retrospective studies are published regarding the safety of this method, but few of the papers were prospective and some publications used this method in low risk patients; high risk patients with suspected adhesions were excluded from the studies.

This study was performed to evaluate safety, ease of insertion, complications and time to get pneumoperitoneum of DTI in various gynecological laparoscopic surgeries.

\section{Materials and Methods}

This is a multicentre retrospective study conducted over a period of six years with effect from $1^{\text {st }}$ April 2012 to $31^{\text {st }}$ March 2018. Total 2814 patients were studied who underwent various laparoscopic surgeries. The study was conducted on patients in whom various gynecological laparoscopic surgeries were performed. Various parameters were recorded are age, BMI, parity, type of surgery, previous scar on the abdomen, time taken to induce pneumoperitoneum and complications in DTI were recorded and analyzed.

In this study all patients were undergoing direct trocar insertion. Written and informed consent was taken for all procedures.

Procedure for Direct Trocar Insertion (DTI): After preparing abdomen small $12 \mathrm{~mm}$, infra-umbilical skin incision was put to stab knife. The anterior abdominal wall was then elevated by the left hand of the surgeon and by assisting surgeon. Then the trocar and cannula with valve open was palmed by the right hand of a surgeon with index finger on cannula to guard. Considerable linear penetration force with screwing movement is generated to propel the sharp, pointed and blind access port directly into the peritoneal cavity, toward the pelvic hollow; as the trocar entered the peritoneal cavity there was hissing sound of air gushing in peritoneal cavity was heard and as air entered the cavity all content fall from the abdominal wall. Then sharp trocar was removed and laparoscope with a light inserted to confirm, correct, safe placement and confirmed any injury. ${ }^{3}$
In the majority of patients the infra-umbilical incision was used. But in patients with previous surgeries supra-umbilical incision was put away from scared area.

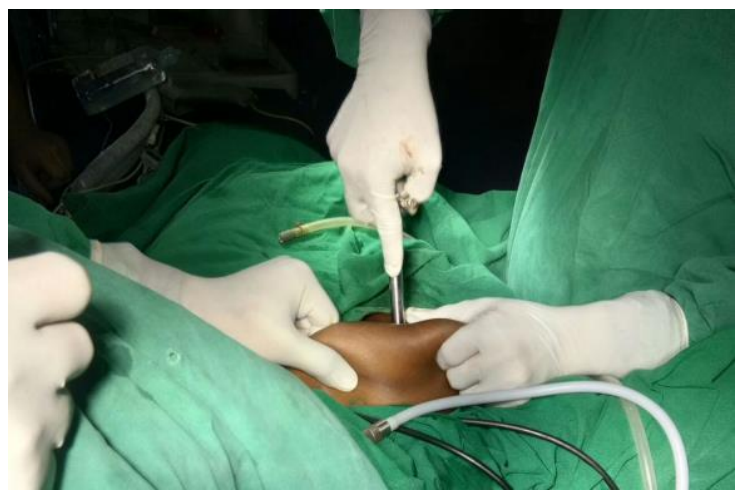

Fig. 1

\section{Result}

Body Mass Index: A patient with higher BMI, having more abdominal fat, DTI is a little bit more difficult. In patients with higher BMI trocar needed to be inserted at an angle of 90 degrees, and it may require more than one attempt to create pneumoperitoneum. And in thin patients direction of trocar was at an angle of 45 degrees.

Table 1

\begin{tabular}{|l|l|}
\hline BMI & \\
\hline Less than 18.5 & $22(0.78 \%)$ \\
\hline $18.5-22.9$ & $1829(65 \%)$ \\
\hline $23-24.9$ & $905(32.2 \%)$ \\
\hline $25-30$ & $38(1.35 \%)$ \\
\hline More than 30 & $20(0.71 \%)$ \\
\hline
\end{tabular}

Parity: In patients with high parity, abdominal wall was lax, so it was easier to insert trocar. In nuliparous patients it was sometime difficult to lift the abdomen as tone of muscle is high. In this case towel clip on both sides of the umbilicus is helpful.

Table 2

\begin{tabular}{|l|c|}
\hline \multicolumn{1}{|c|}{ Parity } & No. of patients \\
\hline Zero & $95(3.37 \%)$ \\
\hline One & $140(4.97 \%)$ \\
\hline Two & $1560(55.43 \%)$ \\
\hline Three or More & $1019(36.21 \%)$ \\
\hline
\end{tabular}

Previous Surgery: In this study total 217 (7.71\%) patients had previous one or more surgeries.

Table 3

\begin{tabular}{|l|l|}
\hline One & $35(1.24 \%)$ \\
\hline Two & $171(6.07 \%)$ \\
\hline Three or more & $11(0.39 \%)$ \\
\hline
\end{tabular}


Type of Laparoscopic Surgery: In this study, $89.48 \%$ patients were for laparoscopic TL, $4.26 \%$ patients were for diagnostic laparoscopy for infertility, $4.69 \%$ patients were for total laparoscopic hysterectomy, $1.5 \%$ patients were for other surgeries.
Table 4

\begin{tabular}{|l|c|}
\hline \multicolumn{1}{|c|}{ Type of surgery } & No. of patients \\
\hline Laparoscopic TL & $2518(89.48 \%)$ \\
\hline Diagnostic laparoscopy & $120(4.26 \%)$ \\
\hline $\begin{array}{l}\text { Total laparoscopic } \\
\text { hysterectomy }\end{array}$ & $132(4.69 \%)$ \\
\hline Laparoscopic cystectomy & $21(0.74 \%)$ \\
\hline Laparoscopic ectopic pregnancy & $19(0.68 \%)$ \\
\hline Laparoscopic vault suspension & $4(0.14 \%)$ \\
\hline
\end{tabular}

Comparison between previous surgeries and no previous surgeries.

Table 5

\begin{tabular}{|l|c|c|c|}
\hline & $\begin{array}{c}\text { Previous surgeries } \\
(\mathbf{N = 2 1 7})\end{array}$ & $\begin{array}{c}\text { No previous } \\
\text { surgery (N=2597) }\end{array}$ & Total (N=2814) \\
\hline Time to induce pneumoperitoneum & 90 second \pm 18 & 80 second \pm 15 & 81 seconds \pm 11 \\
\hline Ability to create pneumoperitoneum & $100 \%, 100 \%$ & $100 \%$ & \\
\hline Abdominal wall hemorrhage & 0 & 0 & 0 \\
\hline Extra peritoneal insufflations & 0 & 0 & 0 \\
\hline Omental injury & $6(2.76 \%)$ & 0 & $6(0.21 \%)$ \\
\hline Major vessel injury & 0 & 0 & 0 \\
\hline Intestinal injury & 0 & 0 & 0 \\
\hline Solid organ injury (uterus) & $1(0.46 \%)$ & 0 & $1(0.036 \%)$ \\
\hline Need for laparotomy & 0 & 0 & 0 \\
\hline
\end{tabular}

In this study successful pneumoperitoneum was established in $100 \%$ patients irrespective of previous surgery, BMI, parity.

Mean time to induce pneumoperitoneum was $81 \pm 11$ seconds in all patients.

Six patients had a minor omental injury as there was omental adhesion to anterior abdominal wall due to previous surgery. These injuries were very small and managed by laparoscopy.

One patient had an injury to uterine fundus. This patient had previous three cesarean deliveries and uterus is adherent to anterior abdominal wall up to fundus and patient had six weeks of pregnancy. Trauma to the uterus was small and managed by bipolar coagulation.

\section{Discussion}

DTI required adequate relaxation, sharp trocar, adequate skin incision, firm elevation of abdominal wall and direction in the pelvis.

Following are the advantages of DTI over other laparoscopic access techniques.

Lifting of the abdominal wall is easy as no pneumoperitoneum was created so the counter force of abdominal wall against the pressure of the tip of tracer is well controlled.

Peritoneal entry is directly confirmed by laparoscope as there is no need of doing various tests for intra-peritoneal access.
It reduces three blind entries (veress needle insertion, pneumoperitoneum creation and trocar insertion) to one blind direct trocar insertion. So mean laparoscope insertion time was reduced significantly from 5 to 7 minutes to 1 to 2 minutes.

As pneumoperitoneum is created after confirmation of peritoneal access by laparoscope there is nil chance of pre-peritoneal or visceral insufflations or gas embolism.

Various studies that favor the direct trocar insertion are as under.

In 1989, Byron et al. used this direct access method in unselected 937 women and recorded 4.2\% complication rate with significant increased risk of minor complications. $2.7 \%$ case had more than three entry attempts. And in $1.4 \%$ cases they failed to access. ${ }^{6}$ He summarized that history of previous abdominal surgery was not associated with an increased risk of access complications. Later they randomized 252 women with veress needle $(n=141)$ and direct trocar access $(\mathrm{n}=111)$; they discover a fourfold increase in minor access complication rates with veress needle group and longer port insertion time of 5.9 vs 2.2 minutes. $^{5}$

Laparoscopic access publications reviewed by Molloy et al. (134917 veress/trocar cannula, 21547 open, 16739 direct entry) and showed visceral-bowel injury rates were $0.04 \%, 0.11 \%$ and $0.05 \%$ respectively, and vessel injury rate were $0.04 \%, 0.01 \%$ and $0 \%$ respectively. ${ }^{7}$ 
Borgatta et al. Studied patients with previous abdominal surgery and showed a two fold increase in omental injury with the veress needle over the direct insertion with longer insertion time of 2 minutes 10 seconds when veress needle closed method was used. ${ }^{8}$

Copeland et al. Reviewed 2000 unselected patients where DTI was used, $0.4 \%$ required conversion to closed veress method and one patient have an inadvertent visceral bowel injury, whereas two $(0.1 \%)$ additional visceral bowel injuries occurred in DTI. ${ }^{9}$

Woolcot r. Studied 6173 gynecological patients and showed $4(0.6 \%)$ perforating bowel injuries and no major vessels injury and gas embolism. ${ }^{10}$

Jacobson MT reviewed 1223 direct trocar insertion and there is no trocar entry related injury in all patients. ${ }^{11}$

Siavash Falahatkar et al. studied 148 patients undergoing urolaparoscopic surgeries; 62 patietns received DTI and 86 patients received open laparoscopy. The mean access time for DTI was 91.75 seconds which was significantly shorter than the mean access time of 263.97 seconds for patients receiving open laparoscopy. There were very few complications in either study group. ${ }^{12}$

Issam Merdan, Sadq Ghleb Kadem \& Yaqoop Ayoob Yaqoop studied 210 patients undergoing various laparoscopic surgeries; 70 (33.33\%) patients operated with veress needle technique, $70(33.33 \%)$ patients operated with DTI technique and 70 (33.33\%) patients operated with modified open trocar insertion technique. The mean time required for entry in patients subjected to veress needle technique was $3.63 \pm 0.64$ minutes in comparison to $1.79 \pm 2.39$ minutes and $2.01 \pm 1.82$ minutes for DTI and modified open trocar insertion respectively. The veress needle technique associated with high rate of minor complications $45.71 \%$ patients in comparison with $7(10 \%)$ patients and $5(7.14 \%)$ patients reported during DTI and modified open trocar insertion respectively. ${ }^{13}$

\section{Conclusion}

My study suggests that DTI is safe, rapid and efficient alternative procedure with low incidence of complications.

\section{References}

1. A manual of minimally invasive gynecological surgery, chapter 2 peritoneal access in laparoscopy.

2. Hasson HM. A modified instrument and method for laparoscopy. Am J Obstet Gynecol. 1971;110:886-7

3. Dingfelder JR. direct laparoscopic trocar insertion without prior pneumoperitoneum. J Reprod Med. 1978;21:45-7.

4. Catarci M, Carlini M, Gentileschi P. Major and minor injuries during the creation of pneumoperitoneum: a multicenter study on 12991 cases. Surg Endos. 2001;15:566-9.

5. Byron JW, Markenson G, Miyazawa K. A randomized comparison of various needles and direct trocar insertion for laparoscopy. Surg Gynecol Obstet. 1993;177:259-62.
6. Byron JW, Fjiyoshi CA, Miyazawa K. Evaluation of the direct trocar insertion technique at laparoscopy. Obstet Gynecol. 1989;74:423-5.

7. Molloy D, Kaloo PD, Cooper M. Laparoscopic entry: a literature review and analysis of techniques and complications of primary port entry. Aust NZJ Obstet Gynecol. 2002;42:246-53.

8. BorgattaL, Gruss L, Barad D. Direct trocar insertion vs veress needle use for laparoscopic sterilization. J Repord MED. 1990;35:891-4.

9. Copeland C, Wing R, Hulka JF, direct trocar insertion at laparoscopy: an evaluation. Obstet Gynecol.1983;62:6559.

10. Woolcot R. The safety of laparoscopy performed by direct trocar insertion and co2 insufflations under vision. Aus NZJ Obstet Gynaecol. 1997;37:216-9.

11. Jacobson MT, Osias J, Bizhang R. The direct trocar technique: an alternative approach to abdominal entry for laparoscopy. J SLS. 2002;6:169-74.

12. Siavash Falahatkar, Direct Trocar Insertion compared with open laparoscopy in patients undergoing urolaparoscopic surgery. Uro Today International Journal. 2009;2(6).

13. Issam Merdan, Sadq Ghleb Kadem \& Yaqoop Ayoob Yaqoop. Safety and efficacy of modified open technique as a first laparoscopic entry in comparison with direct trocar entry and veress needle technique. Basrah Journal of Surgery, December, 22, 2016.

How to cite this article: Patel V. Safety of direct trocar insertion in various gynecological laparoscopic surgeries. Indian J Obstet Gynecol Res. 2018;5(4):485-488. 